\title{
Mechanism associated with aberrant IncRNA MEG3 expression in gestational diabetes mellitus
}

\author{
HAILING ZHANG \\ Department of Antenatal Diagnosis, Weifang People's Hospital, Weifang, Shandong 261041, P.R. China
}

Received October 14, 2018; Accepted May 16, 2019

DOI: $10.3892 /$ etm.2019.8062

\begin{abstract}
Gestational diabetes mellitus (GDM) is a common metabolic condition during pregnancy. Long non-coding RNAs (lncRNAs) have been found to seve critical roles in GDM development; however, the role of lncRNA maternally expressed gene 3 (MEG3) in GDM remains unclear. Therefore, the aim of the present study was to investigate the expression and role of MEG3 in GDM, and to further explore the underlying mechanism. The levels of lncRNA MEG3 in the blood and placental villous tissues of pregnant women with GDM was measured using reverse transcription-quantitative PCR. Bioinformatics analysis and dual luciferase reporter assays were performed to investigate the association between IncRNA MEG3 and microRNA (miR)-345-3p. Transfection was subsequently performed on HTR-8/SVneo cells, a human chorionic trophoblast cell line, to assess the role of lncRNA MEG3 in GDM. In particular, cell viability, cellular migratory/invasive ability and cell apoptosis were analyzed using MTT assay, Transwell assay and flow cytometry, respectively. Compared with pregnant women without GDM, IncRNA MEG3 levels were significantly elevated in the blood and placental villous tissues of GDM pregnant women. miR-345-3p was identified to be a direct target of lncRNA MEG3 using dual luciferase reporter assay, which was found to be reduced in pregnant women with GDM. Further analysis demonstrated that lncRNA MEG3 overexpression significantly inhibited HTR-8/SVneo cell viability, and prevented cell migration and invasion in addition to inducing cell apoptosis. In contrast, lncRNA MEG3 knockdown significantly enhanced HTR-8/SVneo cell viability, promoted cell migration/invasion and reduced cell apoptosis. Inhibiting miR-345-3p expression negated all the observed physiological effects of lncRNA MEG3 knockdown on HTR-8/SVneo cells. In conclusion, lncRNA MEG3 levels were abnormally upregulated in GDM, which participated in the development and progression of GDM by regulating
\end{abstract}

Correspondence to: Dr Hailing Zhang, Department of Antenatal Diagnosis, Weifang People's Hospital, 151 Guangwen Street, Weifang, Shandong 261041, P.R. China

E-mail: zhanghl1809@163.com

Key words: gestational diabetes mellitus, long non-coding RNA maternally expressed gene 3, microRNA-345-3p, HTR-8/SVneo cells human chorionic trophoblast cell physiology. Therefore, lncRNA MEG3 may be a potential diagnostic and therapeutic target for GDM.

\section{Introduction}

Gestational diabetes mellitus (GDM) is a common metabolic condition during pregnancy that is characterized by glucose intolerance, leading to varying degrees of hyperglycemia $(1,2)$. GDM accounts for $>80 \%$ of pregnant women with diabetes, whilst pregnancies complicated by non-gestational diabetes are $<20 \%$ (3). The incidence of GDM varies around the world due to the use of varying diagnostic criteria and environmental factors $(4,5)$. However, incidences of GDM in China had been increasing annually with economic development and changes in lifestyle (6,7). In 2017, 21.3 million or $16.2 \%$ of live births had some form of hyperglycaemia in pregnancy with $\sim 85.1 \%$ due to GDM. (8). The adverse effects of GDM on pregnant women and infants should not be underestimated as hyperglycemia during pregnancy increases the risk of adverse outcomes in the mother and fetus (9). In particular, infants born from pregnant women with GDM are more susceptible to obesity, metabolic and cardiovascular complications during childhood and adulthood $(10,11)$. In addition, $~ 50 \%$ of women with a history of GDM go on to develop type 2 diabetes within five to ten years post-delivery (8). Therefore, GDM research has become a subject of interest in recent years globally.

Currently, oral glucose tolerance test is a commonly used clinical diagnostic tool for GDM. However, this test is usually performed between 24 and 28 or 32 weeks of gestation (12). This timeframe restricts pregnant women from altering diet and exercise regimens, or to access medical interventions; hence increasing the risk of GDM in late pregnancy. Therefore, there is an urgent demand for developing novel diagnostic approaches for the accurate and rapid prediction of GDM.

Long non-coding RNA (lncRNAs) is a type of functional RNA molecule consisting of $>200$ nucleotides, which lacks the ability to encode proteins (13). LncRNAs can regulate protein-coding genes in a number of ways, including forming complexes with target genes, as well as promoting the degradation and inhibiting the translation and modification of target microRNAs (miRNA). LncRNAs can regulate the expression of genes at epigenetic, transcriptional and post-transcriptional levels, and are widely involved in most physiological and pathological processes in the body (14). Studies have suggested 
that the aberrant expression of IncRNAs is closely associated with the development of complex diseases, including cancer, cardiovascular diseases, nervous system diseases and diabetes (15-18).

LncRNA maternally expressed gene 3 (MEG3) is a known imprinted gene that is $\sim 1.6 \mathrm{kbp}$ in length and located on human chromosome 14q32.2 (19). Imprinting of this gene is controlled by upstream intergenic differentially methylated region (20). In recent years, a number of studies have demonstrated that lncRNA MEG3 is involved in the development and progression of diseases, including retinopathy, osteoarthritis and cardiovascular disease (21-23). It was previously found that lncRNA MEG3 may serve a role in micro-vascular dysfunction that is associated with diabetes (24). In addition, the upregulation of IncRNA MEG3 promotes insulin resistance in the liver by increasing forkhead box $\mathrm{O} 1$ expression (25). In mouse pancreatic $\beta$-cells, lncRNA MEG3 promotes the expression of v-maf musculoaponeurotic fibrosarcoma oncogene family protein $\mathrm{A}$ and affects insulin production by inhibiting the expression of RAD21 cohesin complex component, structural maintenance of chromosomes 3 or transcriptional regulator SIN3A (26). These studies indicate that IncRNA MEG3 is closely associated with insulin resistance. However, the role of lncRNA MEG3 in the development of GDM and its potential molecular mechanism remain unclear. Therefore, the present study aimed to investigate the expression and role of lncRNA MEG3 in GDM, and to explore any potential underlying mechanism.

\section{Materials and methods}

Clinical samples. A total of 20 paired blood samples and matched placental villous tissues were collected from pregnant women between 23 and 37 years old with or without GDM at Weifang People's Hospital (Weifang, China) between May 2015 and May 2017. Fasting peripheral blood was collected from all pregnant women following 28 weeks of gestation, whilst the placental tissues were obtained after delivery. Pregnant women with the following conditions were excluded from the present study: i) Abnormal blood lipid (triglyceride, low-density lipoprotein cholesterol and high-density lipoprotein cholesterol) levels, hypertension, and chronic liver and kidney diseases; ii) endocrine diseases, including, thyroid disease, adrenal cortical disease, obesity, osteoporosis, diabetes and hyperthyroidism prior to pregnancy; iii) pregnant women currently undergoing long-term drug treatments (such as sodiumlevothyroxine) that affect the metabolism of carbohydrates; and iv) other pregnancy complications (pregnancy-induced hypertension, pre-eclampsia, pregnancy with chronic nephritis). GDM was diagnosed if the fasting plasma glucose (FPG) level was $\geq 5.1 \mathrm{mmol} / 1$. GDM was excluded if the FPG level was $\leq 4.4 \mathrm{mmol} / \mathrm{l}$. Women with an FPG level $\geq 4.4 \mathrm{mmol} / 1$ but $\leq 5.1 \mathrm{mmol} / 1$ underwent a $75 \mathrm{~g}$ oral glucose tolerance test (OGTT). In such cases, a diagnosis of GDM was made when at least one glucose value was elevated (FPG $\geq 5.1 \mathrm{mmol} / 1,1-\mathrm{h}$ OGTT $\geq 10.0 \mathrm{mmol} / 1$ or 2 -h OGTT $\geq 8.5 \mathrm{mmol} / \mathrm{l})$. Inclusion criteria: Women with DGM who did not fulfill any of the exclusion criteria were involved in the present study. Written informed consent was obtained from each patient and the present study was approved by the Ethics Committee of Weifang People's Hospital.
Cell culture. HTR-8/SVneo cells, a human chorionic trophoblast cell line, were purchased from Shanghai Huzhen Industrial Co., Ltd. (cat. no. HZ-CC337655). Cells were cultured in RPMI 1640 medium (Gibco; Thermo Fisher Scientific, Inc.) supplemented with 10\% FBS (Gibco; Thermo Fisher Scientific, Inc.) and 1\% streptomycin-penicillin solution (Beyotime Institute of Biotechnolohy), and incubated in a humidified atmosphere under $5 \% \mathrm{CO}_{2}$ at $37^{\circ} \mathrm{C}$.

Cell transfection. The MEG3 sequence was synthesized based on the MEG3 sequence and then sub-cloned into the pcDNA3.1 vector (pcDNA-MEG3; Shanghai GeneChem Co., Ltd.). The empty pcDNA3.1 vector was used as a control (pcDNA-control). For MEG3 knockdown, the MEG3-shRNA plasmid (sh-MEG3 sequence, 5'-GAGAGGTTGTTT CACTGGTATCTATTGCA-3'; pGFP-C-shLenti Vector; cat. no. TL 320132C) and the scrambled shRNA plasmid (sh-control; pGFP-C-shLenti Vector; cat. no. TR30021) were purchased from OriGene. HTR-8/SVneo cells were seeded into 6 -well plates $\left(1 \times 10^{6}\right.$ cells/well) and cultured at $37^{\circ} \mathrm{C}$ for $24 \mathrm{~h}$. The cells were subsequently transfected with $100 \mathrm{ng}$ pcDNA-control, $100 \mathrm{ng}$ pcDNA-MEG3, $100 \mathrm{ng}$ sh-control, $100 \mathrm{ng}$ sh-MEG3, $100 \mathrm{nM}$ miR-345-3p inhibitor (cat. no. HmiR-AN0437-AM01; GeneCopoeia, Inc.), 100 nM inhibitor control (cat. no. CmiR-AN0001-SN; GeneCopoeia, Inc.) or $100 \mathrm{ng}$ sh-MEG3 + $100 \mathrm{nM}$ miR-345-3p inhibitor using Lipofectamine ${ }^{\circledR} 2000$ reagent (Invitrogen; Thermo Fisher Scientific, Inc.) according to the manufacturer's protocols. Transfection efficiency was assessed using reverse transcription-quantitative PCR (RT-qPCR) $48 \mathrm{~h}$ after transfection.

Bioinformatics analyses. Bioinformatics analysis (http://starbase.sysu.edu.cn/index.php) (27) was performed to predict potential binding sites between lnc-MEG3 and miR-345-3p using the 'miRNA-lncRNA' search function.

Luciferase reporter assay. The putative binding sequences of MEG3-wild type (WT; 5'-CCAGAGCCTGGTTCA GGG-3') and MEG3-mutant (MUT; 5'-AACCCGAAGGCG GACAAA-3') were respectively cloned into a pmirGLO vector (Promega Corporation). HTR-8/SVneo cells were co-transfected with $50 \mathrm{nM}$ either miR-345-3p mimics (HmiR0210-MR03; GeneCopoeia, Inc.) or mimic control (cat. no. CmiR0001-MR03; GeneCopoeia, Inc.) and 100 ng either MEG3-WT or MEG3-MUT using Lipofectamine ${ }^{\circledR} 2000$. Following $48 \mathrm{~h}$ transfection, Dual-Luciferase Reporter Assay System (Promega Corporation) was used to detect luciferase activity, according to the manufacturer's protocols. All firefly luciferase activities were normalized to Renilla luciferase activity.

Cell viability assay. HTR-8/SVneo cell viability (1x10 ${ }^{4}$ cells/well) was measured using MTT assay. Following $48 \mathrm{~h}$ transfection, $20 \mu \mathrm{l}$ MTT solution $(0.5 \mathrm{mg} / \mathrm{ml})$ was added into each well. After $4 \mathrm{~h}$ incubation at $37^{\circ} \mathrm{C}, 150 \mu \mathrm{l}$ dimethyl sulfoxide (DMSO) (Sigma-Aldrich; Merck KGaA) was used to dissolve the formazan crystals. Finally, cell viability was detected by determining absorbance values at $570 \mathrm{~nm}$ using a FLUOstar ${ }^{\circledR}$ Omega Microplate Reader (BMG Labtech $\mathrm{GmbH})$. 
Apoptosis analysis. Annexin V-fluorescein isothiocyanate (FITC)/propidium iodide (PI) apoptosis detection kit [cat. no. 70-AP101-100; Multisciences (Lianke) Biotech Co., Ltd.] was used to assess cell apoptosis. Briefly, $48 \mathrm{~h}$ after cell transfection, HTR-8/SVneo cells $\left(1 \times 10^{6}\right)$ were collected though centrifugation $(1,000 \mathrm{xg})$ at $4^{\circ} \mathrm{C}$ for $5 \mathrm{~min}$, then the cells were stained using $5 \mu \mathrm{l}$ Annexin V-FITC and $5 \mu \mathrm{l} \mathrm{PI}$ for $30 \mathrm{~min}$ at room temperature in the dark. Lastly, apoptotic cells were analyzed using a flow cytometer (BD Biosciences) with WinMDI soft-ware (version 2.5; Purdue University Cytometry Laboratories; www.cyto.purdue. edu/flowcyt/software/Catalog.htm).

Transwell assay. The invasive and migratory abilities of HTR-8/SVneo cells were measured using Transwell inserts (Corning Inc.) with or without Matrigel (BD Biosciences), respectively. For cell invasion assay, Transwell inserts were pre-coated with Matrigel and incubated at $37^{\circ} \mathrm{C}$ for $5 \mathrm{~h}$. Following $48 \mathrm{~h}$ transfection, HTR-8/SVneo cells $\left(2 \times 10^{4}\right)$ suspended in $100 \mu \mathrm{l}$ serum-free medium were seeded into the upper chamber, whilst $500 \mu 1$ medium supplemented with $20 \%$ FBS was added into the lower chamber. The cells were then incubated for 48 -h at $37^{\circ} \mathrm{C}$. The migratory or invasive cells were subsequently fixed with $100 \%$ methanol at room temperature for $20 \mathrm{~min}$, stained with $0.1 \%$ crystal violet at $37^{\circ} \mathrm{C}$ for $20 \mathrm{~min}$ and counted under an inverted light microscope (magnification, x100) in five randomly-selected fields.

RNA extraction and RT-qPCR. Total RNA was extracted from blood, tissues and cells using the TRIzol reagent (Thermo Fisher Scientific, Inc.) according to manufacturer's protocols and the cDNA of miR-345-3p was synthesized using the TaqMan ${ }^{\mathrm{TM}}$ miRNA reverse transcription kit (Thermo Fisher Scientific, Inc.) according to manufacturer's protocols. The temperature protocol for the reverse transcription reaction consisted of primer annealing at $25^{\circ} \mathrm{C}$ for 5 min, cDNA synthesis at $42^{\circ} \mathrm{C}$ for $60 \mathrm{~min}$ and termination at $80^{\circ} \mathrm{C}$ for $2 \mathrm{~min}$. miR-345-3p levels were quantified using TaqMan ${ }^{\mathrm{TM}}$ human MiRNA assay kit (Thermo Fisher Scientific, Inc.) according to manufacturer's protocols. cDNA synthesis and subsequent qPCR of lncRNA MEG3 were performed using SYBR ${ }^{\circledR}$ Premix Ex Taq ${ }^{\mathrm{TM}}$ (Tli RNaseH Plus; Takara Biotechnology Co., Ltd.) according to manufacturer's protocols. The following thermocycling conditions were used for PCR: Initial denaturation at $95^{\circ} \mathrm{C}$ for $45 \mathrm{sec}$, followed by 40 cycles of $95^{\circ} \mathrm{C}$ for $10 \mathrm{sec}$ and $52^{\circ} \mathrm{C}$ for $35 \mathrm{sec}$. U6 and GAPDH were used as the endogenous controls for miR-345-3p and lncRNA MEG3, respectively. The primer sequences used for qPCR were as follows: lncRNA MEG3 forward, 5'-CTG CCCATCTACACCTCACG-3' and reverse, 5'-CTCTCCGCC GTCTGCGCTAGGGGCT-3'; GAPDH forward, 5'-CTTTGG TATCGTGGAAGGACTC-3' and reverse, 5'-GTAGAGGCA GGGATGATGTTCT-3'; U6 forward, 5'-GCTTCGGCAGCA CATATACTAAAAT-3' and reverse, 5'-CGCTTCACGAAT TTGCGTGTCAT-3'; miR-345-3p forward, 5'-GGTTTTTGG ATTGGGTTGTAGAGTG-3' and reverse, 5'-AACCAAAAC AATCCCTTACCACTAC-3'. Relative gene expression was calculated using the $2^{-\Delta \Delta \mathrm{Cq}}$ method (28).

Western blotting. Total protein was extracted from cells using the radioimmunoprecipitation assay buffer kit (Thermo Fisher
Scientific, Inc.). Protein concentration was quantified using a bicinchoninic acid assay kit (Thermo Fisher Scientific, Inc.) prior to separation via SDS-PAGE on a $10 \%$ gel at $30 \mu \mathrm{g}$ protein/lane. The separated proteins were then transferred onto polyvinylidene fluoride membranes and blocked with $5 \%$ non-fat milk at room temperature for $1 \mathrm{~h}$. Following blocking, the membranes were then incubated with primary antibodies against Bcl-2 (cat no. 4223), Bax (cat no. 5023) and $\beta$-actin (cat no. 4970; all dilution: 1:1,000; Cell Signaling Technology, Inc.) at $4^{\circ} \mathrm{C}$ overnight. The membranes were washed with phosphate buffer saline (PBS)-0.05\% Tween 205 times and then incubated with a horseradish peroxidase-conjugated anti-rabbit immunoglobulin G secondary antibody (cat no. 7074; dilution: 1:2,000; Cell Signaling Technology, Inc.) at room temperature for $2 \mathrm{~h}$. The protein bands were visualized using an enhanced chemiluminescence kit (Applygen Technologies, Inc.) according to manufacturer's protocols. Densitometry was performed using the ImageJ software (version 1.38X; National Institutes of Health).

Statistical analysis. Data were presented as the mean \pm SD. Statistical analysis was performed using the SPSS software (version 18.0; SPSS, Inc.). Differences between multiple groups were analyzed using one-way ANOVA followed by Tukey's post-hoc test, and differences between two groups were analyzed using Student's t-test. $\mathrm{P}<0.05$ was considered to indicate a statistically significant difference.

\section{Results}

LncRNA MEG3 is highly expressed in blood and placental villous tissues of pregnant women with GMD. The lncRNA MEG3 levels in the blood and placental villous tissues of pregnant women were measured using RT-qPCR. LncRNA MEG3 levels in the blood and placental villous tissues from pregnant women with GDM were significantly upregulated compared with those without GDM (Fig. 1A and B). This suggest that lncRNA MEG3 may serve a role during GDM.

miR-345-3p is a target of lncRNA MEG3. Bioinformatics analysis (http://starbase.sysu.edu.cn/index.php) showed miR-345-3p may be a target of lncRNA MEG3 (Fig. 2A). Luciferase assay was used to confirm whether lncRNA MEG3 could regulate miR-345-3p expression by acting as molecular sponge. Luciferase activity was significantly reduced in cells co-transfected with the miR-345-3p mimic and MEG3-WT compared with cells co-transfected with mimic control (miR-control) and MEG3-WT. However, no significant differences were observed between the luciferase activity of cells co-transfected with mimic control (miR-control) and MEG3-MUT and that in cells co-transfected with the miR-345-3p mimic and MEG3-MUT (Fig. 2B). This finding suggests that miR-345-3p is a direct target of lncRNA MEG3.

miR-345-3p is downregulated in the blood and placental villous tissues of pregnant women with GMD. The levels of miR-345-3p in the blood and placental villous tissues of pregnant women with or without GMD were next measured using RT-qPCR. miR-345-3p levels in the blood (Fig. 3A) and placental villous tissues (Fig. 3B) of women with GMD was 

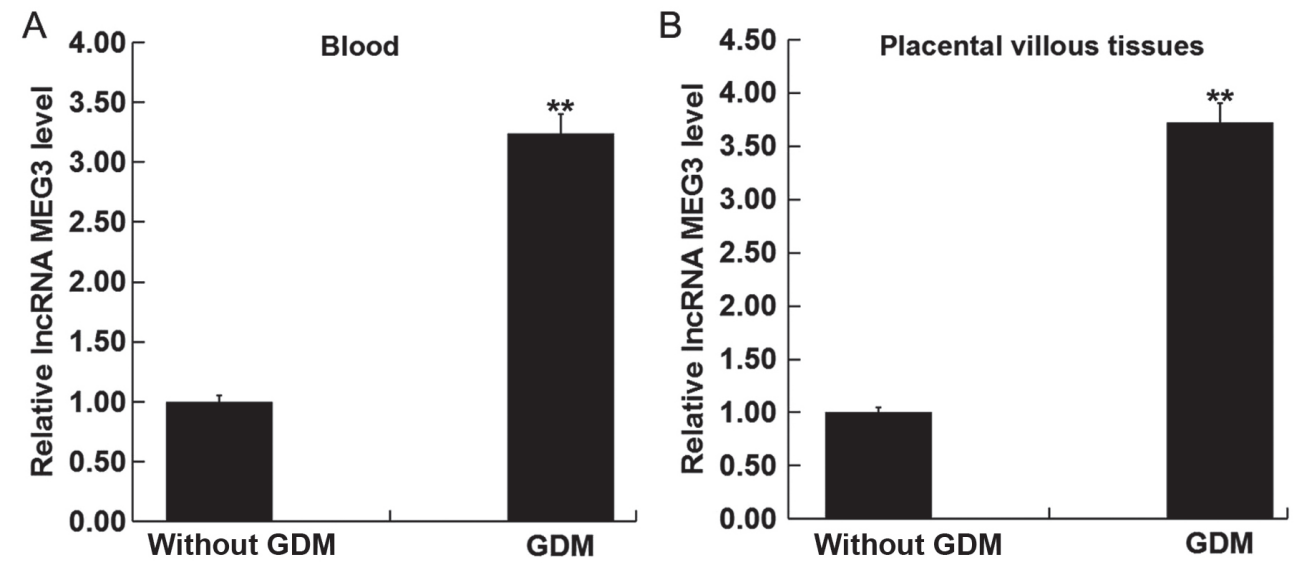

Figure 1. LncRNA MEG3 expression in GDM pregnant women. LncRNA MEG3 levels in the (A) blood and (B) placental villous tissues of pregnant women with or without GDM by using reverse transcription-quantitative PCR. Data represent the mean $\pm \mathrm{SD}$. ${ }^{* *} \mathrm{P}<0.01$ vs. Without GDM. LncRNA, long non-coding RNA; MEG3, maternally expressed gene 3; GDM, gestational diabetes mellitus.

A $\begin{array}{cc}\text { miR-345-3p } & \text { 3'-gaGGUCUGGGGAGCAAGUCCCg-5' } \\ & \|\|\|\|\|\|\|\|\|\| \\ \text { MEG3 WT } & \text { 5'-ccCCAGA-GCCTGGTTCAGGG-3' } \\ \text { MEG3 MUT } & \text { 5'-ccGGCCC - GGGAGCAAGCCCCC-3' }\end{array}$

B

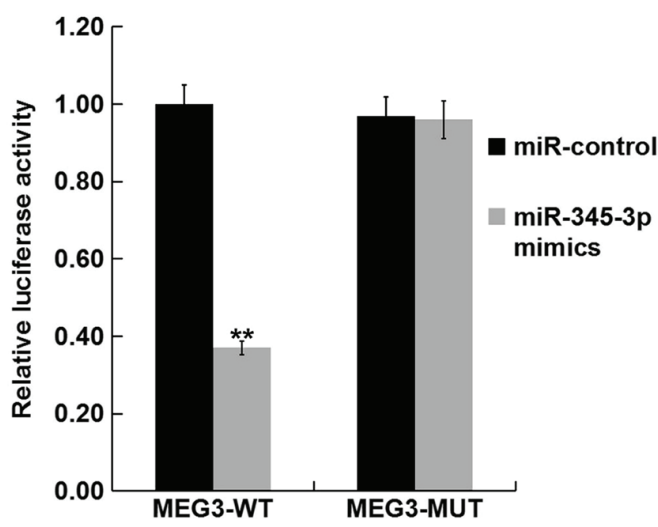

Figure 2. miR-345-3p is a target of 1ncRNA MEG3. (A) Potential lncRNA MEG3 binding sites on the miR-345-3p sequence. (B) Relative luciferase activity of a reporter encoding either IncRNA MEG3 3'-UTR WT or IncRNA MEG3 3'-UTR MUT in HTR-8/SVneo cells co-transfected with either miR-control or miR-345-3p mimics. All data represent the mean \pm SD from three independent experiments. ${ }^{* *} \mathrm{P}<0.01$ vs. miR-control. miR, microRNA; 3'-UTR, 3'-untranslated region; lncRNA, long non-coding RNA; MEG3, maternally expressed gene 3; WT, wild type; MUT, mutant; GDM, gestational diabetes mellitus.

significantly reduced compared with pregnant women without GDM.

LncRNA MEG3 overexpression inhibits viability, migration and invasion of placental trophoblast cells, and induces apoptosis. The effects of lncRNA MEG3 overexpression on human chorionic trophoblast cells (HTR-8/SVneo cells) was next investigated by transfecting HTR-8/SVneo cells with pcDNA-control or pcDNA-MEG3. Following 48-h transfection, efficiency was evaluated using RT-qPCR. pcDNA-MEG3 transfection significantly increased the levels of IncRNA MEG3 in HTR-8/SVneo cells compared with cells in the control group and pcDNA control group (Fig. 4A). HTR-8/SVneo cell viability, apoptosis, migratory and invasive ability were subsequently analyzed using MTT, flow cytometry and Transwell assays, respectively. LncRNA MEG3 overexpression significantly inhibited HTR-8/SVneo cell viability (Fig. 4B), and prevented cell migration (Fig. 4C) and invasion (Fig. 4D), in addition to significantly inducing cell apoptosis (Fig. 4E and F) compared with cells in the control group and pcDNA control group. Supporting this, it was also found that lncRNA MEG3 overexpression significantly decreased protein levels of Bcl-2 whilst increasing levels of Bax compared with the control group and pcDNA control group (Fig. 4G-I).

LncRNA MEG3 knockdown promotes the viability, migration and invasion of placental trophoblast cells, and reduces apoptosis. The effects of lncRNA MEG3 knockdown on HTR-8/SVneo cells was next investigated. HTR-8/SVneo cells were transfected with sh-control, sh-MEG3, inhibitor control, miR-345-3p inhibitor or sh-MEG3 + miR-345-3p inhibitor for $48 \mathrm{~h}$ prior to RT-qPCR analysis to assess transfection efficiency. Compared with the control group and sh-control group, sh-MEG3 significantly reduced the levels of lncRNA MEG3 in HTR-8/SVneo cells (Fig. 5A), whereas the miR-345-3p inhibitor significantly reduced miR-345-3p levels in HTR-8/SVneo cells compared with the control group and inhibitor control group (Fig. 5B). sh-MEG3 significantly increased the levels of miR-345-3p in the HTR-8/SVneo cells compared with the control group and sh-control group, which was partially reversed by transfection with the miR-345-3p inhibitor (Fig. 5C).

Results from MTT assay, Transwell assay, and flow cytometry demonstrated that lncRNA MEG3 knockdown significantly promoted HTR-8/SVneo cell viability (Fig. 6A), enhanced cell migration and invasion (Fig. 6B and C) and reduced cell apoptosis (Fig. 6D and E) compared with the control group and sh-control group. In addition, compared with the control group and sh-control group, lncRNA MEG3 knockdown significantly increased Bcl-2 protein expression whilst reducing Bax protein expression (Fig. 6F-H). All the observed effects that lncRNA MEG3 knockdown exerted on HTR-8/SVneo cells were negated by transfection with the miR-345-3p inhibitor (Fig. 6). 

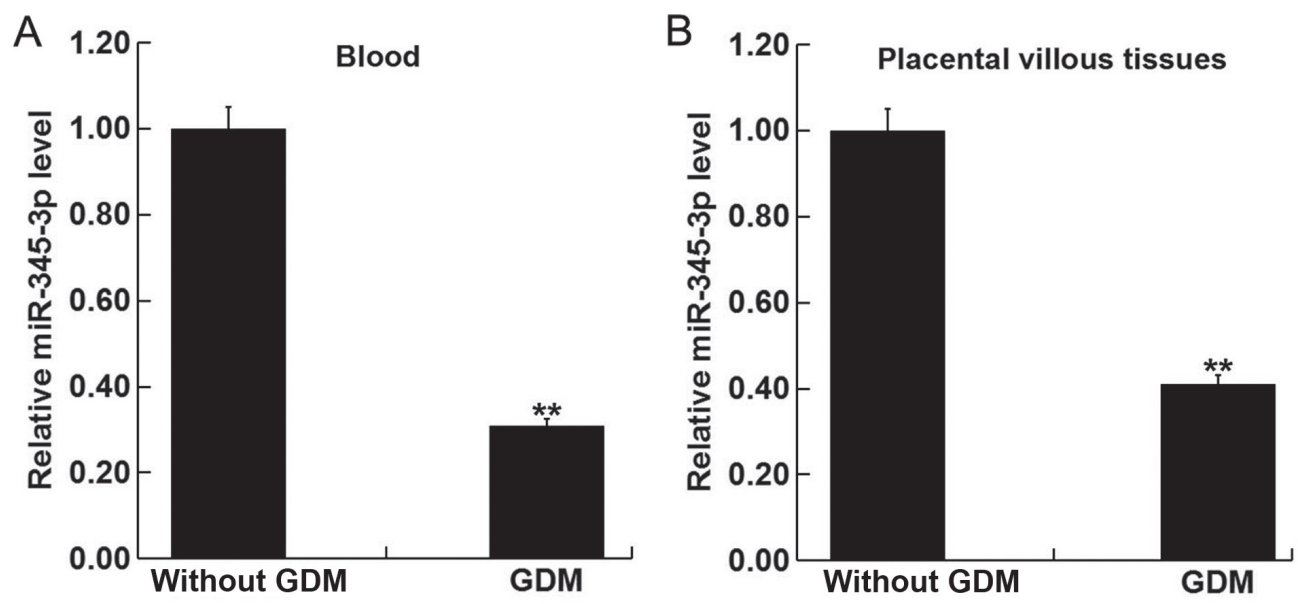

Figure 3. miR-345-3p expression in pregnant women with GDM. The levels of miR-345-3p in the (A) blood and (B) placental villous tissues of pregnant women with or without GDM using reverse transcription-quantitative PCR. Data represent the mean $\pm \mathrm{SD}$. ${ }^{* *} \mathrm{P}<0.01$ vs. Without GDM. miR, microRNA; GDM, gestational diabetes mellitus.
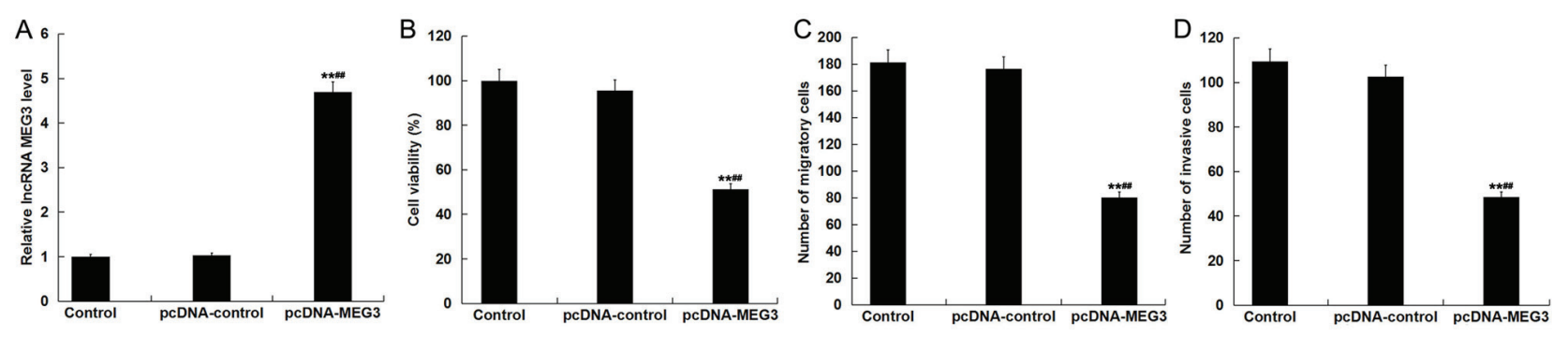

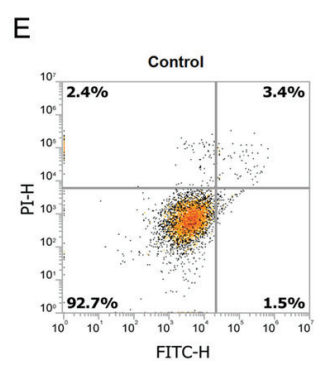

G

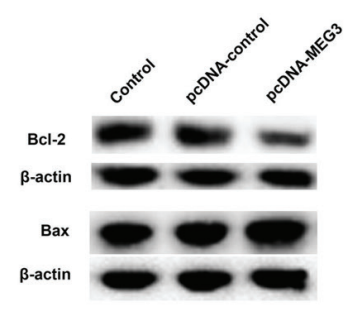

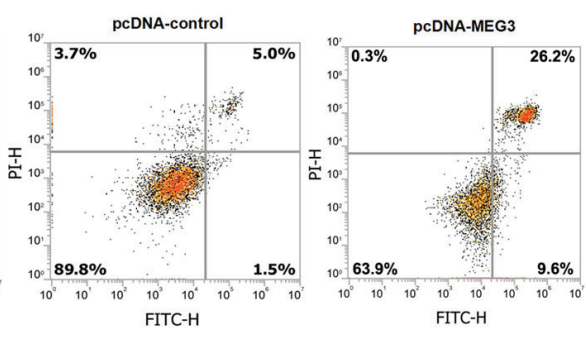

$\mathrm{H} 0.80$

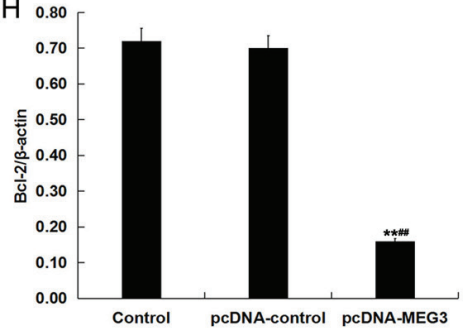

F 40
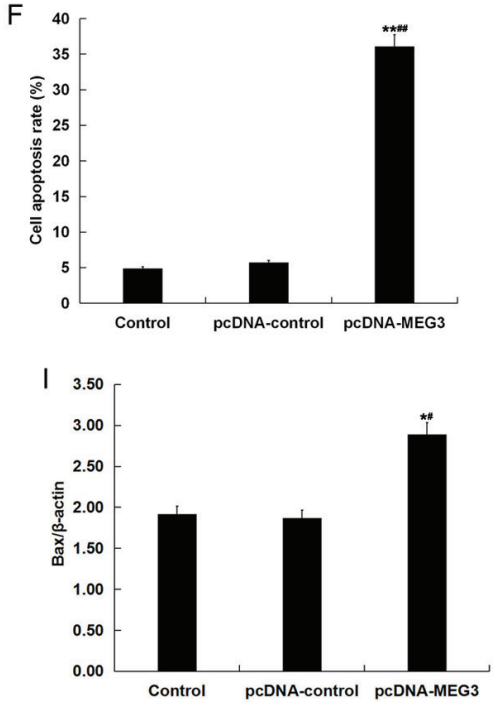

Figure 4. Effect of 1ncRNA MEG3 overexpression on HTR-8/SVneo cells. (A) The levels of 1ncRNA MEG3, (B) cell viability, (C) number of migratory cells and (D) number of invasive cells in HTR-8/SVneo cells transfected with pcDNA-control or pcDNA-MEG3. (E and F) Cell apoptosis was measured in HTR-8/SVneo cells transfected with pcDNA-control or pcDNA-MEG3 using flow cytometry. (G-I) Protein expression levels of (H) Bcl-2 and (I) Bax in HTR-8/SVneo cells transfected with pcDNA-control or pcDNA-MEG3. Data represent the mean $\pm \mathrm{SD}$, (magnification, $\mathrm{x} 200)$. ${ }^{*} \mathrm{P}<0.05$ and ${ }^{* *} \mathrm{P}<0.01 \mathrm{vs}$. Control (untransfected cells); ${ }^{\#} \mathrm{P}<0.05$ and ${ }^{\# \#} \mathrm{P}<0.01$ vs. pcDNA-control. LncRNA, long non-coding RNA; MEG3, maternally expressed gene 3; GDM, gestational diabetes mellitus; FITC, fluorescein isothiocyanate; PI, propidium iodide.

\section{Discussion}

The present study demonstrated that the levels of lncRNA MEG3 in the blood and placental villous tissues from pregnant women with GDM was significantly upregulated compared with the pregnant women without GDM. miR-345-3p was found to be a target of IncRNA MEG3, which was downregulated in the blood and placental villous tissues from pregnant women with GMD. LncRNA MEG3 overexpression inhibited the cell viability, migration and invasion in placental trophoblast cells, and induced apoptosis, whilst suppression of lncRNA MEG3 expression exhibited the opposite effects. In 

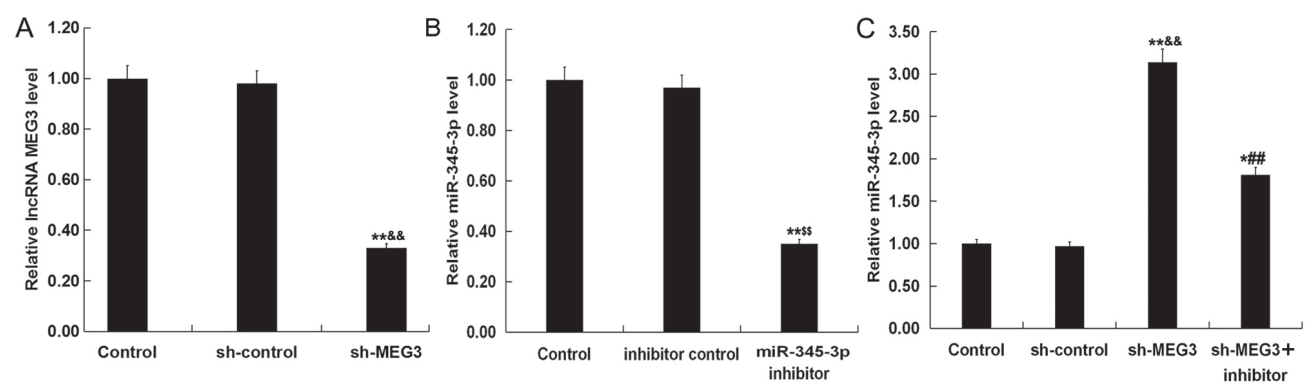

Figure 5. Expression of lncRNA MEG3 and miR-345-3p in HTR-8/SVneo cells. The level of (A) lncRNA MEG3 and (B) miR-345-3p in HTR-8/SVneo cells transfected with miR-345-3p inhibitor or inhibitor control. (C) The levels of miR-345-3p in HTR-8/SVneo cells transfected with sh-control, sh-MEG3 or miR-345-3p inhibitor + sh-MEG3. Data represent the mean $\pm \mathrm{SD}$. ${ }^{*} \mathrm{P}<0.05$ and ${ }^{* *} \mathrm{P}<0.01$ vs. Control; ${ }^{\# \#} \mathrm{P}<0.01$ vs. sh-MEG3; ${ }^{\& \&} \mathrm{P}<0.01$ vs. sh-control; ${ }^{\text {s\$ }} \mathrm{P}<0.01$ vs. inhibitor control. sh, short hairpin RNA; lncRNA, long non-coding RNA; MEG3, maternally expressed gene 3; miR, microRNA.
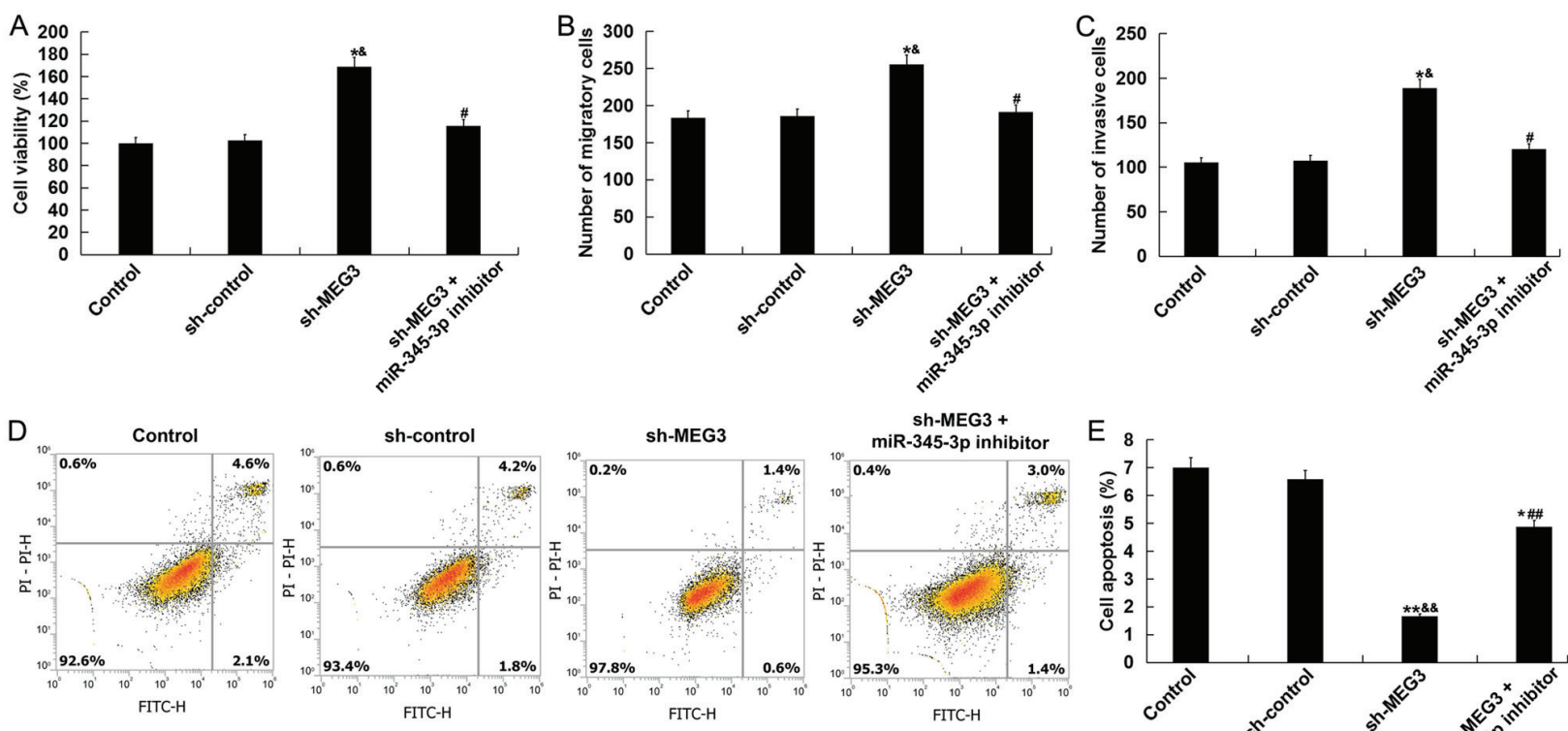

$\mathrm{F}$
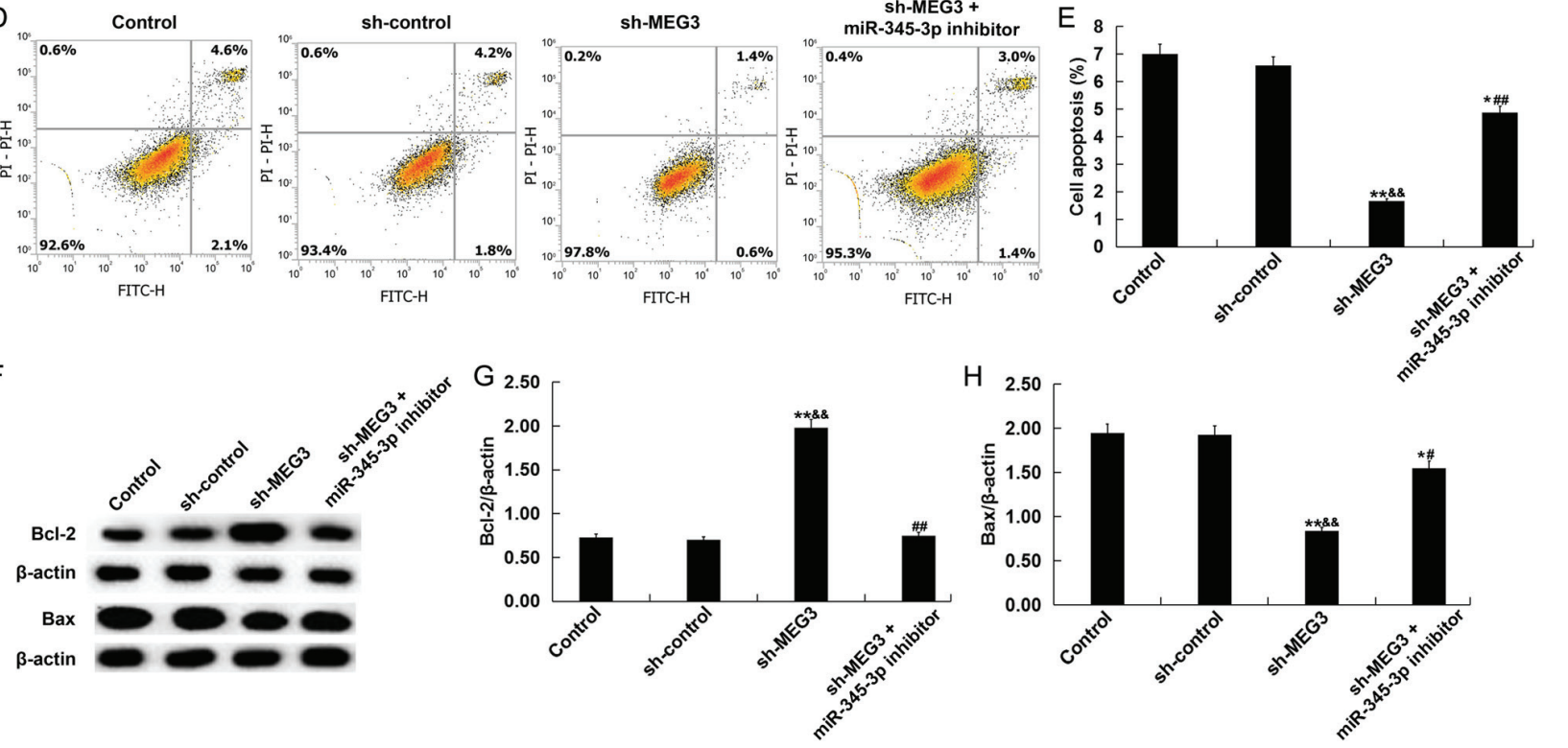

Figure 6. Effect of lncRNA MEG3 and miR-345-3p downregulation on HTR-8/SVneo cells. (A) Cell viability, (B) the number of migratory cells, (C) the number of invasive cells, (D and E) cell apoptosis and (F-H) Bcl-2 and Bax protein expression in HTR-8/SVneo cells transfected with sh-control, sh-MEG3 or miR-345-3p inhibitor + sh-MEG3. Data represent the mean $\pm \mathrm{SD}$, (magnification, $\mathrm{x} 200$ ). ${ }^{*} \mathrm{P}<0.05$ and ${ }^{* *} \mathrm{P}<0.01$ vs. Control; ${ }^{\#} \mathrm{P}<0.05$ and ${ }^{\# \#} \mathrm{P}<0.01$ vs. sh-MEG3. ${ }^{\&} \mathrm{P}<0.05$ and ${ }^{\& \&} \mathrm{P}<0.01$ vs. sh-control. LncRNA, long non-coding RNA; MEG3, maternally expressed gene 3; GDM, gestational diabetes mellitus.

addition, all the effects of lncRNA MEG3 knockdown exerted on HTR-8/SVneo cells were reversed by the inhibition of miR-345-3p expression. These findings suggest that lncRNA MEG3 may be a novel diagnostic biomarker and therapeutic target for GDM.

Aberrant expression of lncRNAs is closely associated with the development of complex diseases, including cancer, cardiovascular diseases, nervous system diseases and diabetes (16-19). IncRNA MEG3 has been previously reported to participate in the development and progression of diseases, including retinopathy, osteoarthritis and cardiovascular disease (21-23). In particular, inhibition of DNA methyltransferase 3b (DNMT3B) has been shown to upregulate MEG3 expression in HCC cells (29). In recent years, a number of studies have suggested that lncRNAs participate in the occurrence and development of GDM $(30,31)$. LncRNA MEG3, which is closely associated with insulin resistance in the body, is involved in the occurrence and development 
of diabetes (21-23). The pathogenesis of GDM is highly analogous to that of type 2 diabetes $(32,33)$. Therefore, it was hypothesized in the present study that lncRNA MEG3 may serve an important role in the development of GDM.

Firstly, the expression of lncRNA MEG3 in the blood and placental tissues from pregnant women with GDM and non-GDM was examined. LncRNA MEG3 was demonstrated to be highly expressed in the blood and placental villous tissues from pregnant women with GDM. It was subsequently found that miR-345-3p was a target of 1ncRNA MEG3. miR-345-3p expression has been reported to be downregulated in diabetes mellitus (34) and diabetic cardiomyopathy (35). In the present study, it was found that miR-345-3p was downregulated in the blood and placental villous tissues from pregnant women with GMD. These observations suggested an important role for lncRNA MEG3 in GDM.

Placental trophoblasts serve an important role in the process of blastocyst implantation in early pregnancy $(36,37)$. Trophoblast proliferation, apoptosis, invasion and migration are key to the establishment, maintenance and finally timely termination of physiological pregnancy $(37,38)$. In particular, placental trophoblast function has become the focus of intense research on GDM pathogenesis (39). Therefore, the effect of lncRNA MEG3 on human chorionic trophoblast cell physiology was next investigated using the HTR-8/SVneo cell line as model. LncRNA MEG3 overexpression inhibited HTR-8/SVneo cell viability, migration and invasion in addition to inducing apoptosis; whilst the suppression of lncRNA MEG3 expression exerted the opposite effects. Notably, the effects lncRNA MEG3 knockdown exerted on HTR-8/SVneo cells were negated by miR-345-3p downregulation. However, a limitation of the present study was that a sh-MEG3 + inhibitor control group was not evaluated.

Taken together, results from the present study suggest that the levels of lncRNA MEG3 was significantly upregulated in GDM, and it participated in the development and progression of GDM. This was possibly mediated via the regulation of human chorionic trophoblast cell physiology by targeting miR-345-3p expression. Therefore, lncRNA MEG3 may be a diagnostic and therapeutic target for GDM. However, the present study is only a preliminary study on the role of lncRNA MEG3 in GDM in vitro. To enhance the scientific significance of the results from the present study, the relationship between the levels of lncRNA MEG3 and blood glucose levels should be determined; in addition to in vivo studies on the role of lncRNA MEG3 in GDM development. These issues should be addressed further in any future research.

\section{Acknowledgements}

Not applicable.

\section{Funding}

No funding was received.

\section{Availability of data and materials}

All data sets used and/or generated during the present study are available from the corresponding author on reasonable request.

\section{Authors' contributions}

HZ designed the study, performed all the experiments and sample collection, analyzed the data and prepared the manuscript.

\section{Ethics approval and consent to participate}

Written informed consent was obtained from each patient and this study was approved by the Ethics Committee of Weifang People's Hospital (Weifang, China). Written informed consent was obtained from each patient.

\section{Patient consent for publication}

Not applicable.

\section{Competing interests}

The author declares that they have no competing interests.

\section{References}

1. Surapaneni T, Nikhat I and Nirmalan PK: Diagnostic effectiveness of $75 \mathrm{~g}$ oral glucose tolerance test for gestational diabetes in India based on the international association of the diabetes and pregnancy study groups guidelines. Obstet Med 6: 125-128, 2013.

2. Djelmis J, Pavic M, Mulliqi Kotori V, Pavlić Renar I, Ivanisevic M and Oreskovic S: Prevalence of gestational diabetes mellitus according to IADPSG and NICE criteria. Int J Gynaecol Obstet 135: 250-254, 2016.

3. Liao EY: Endocrine and metabolic diseases. Beijing. People's Medical Publishing House. 2012.5 ISBN: 9787117151153. https:// baike.so.com/doc/

4. Egan AM, Vellinga A, Harreiter J, Simmons D, Desoye G, Corcoy R, Adelantado JM, Devlieger R, Van Assche A, Galjaard S, et al: Epidemiology of gestational diabetes mellitus according to IADPSG/WHO 2013 criteria among obese pregnant women in Europe. Diabetologia 60: 1913-1921, 2017

5. Song L, Shen L, Li H, Liu B, Zheng X, Zhang L, Xu S and Wang Y: Socio-economic status and risk of gestational diabetes mellitus among Chinese women. Diabet Med 34: 1421-1427, 2017.

6. Zhang F, Dong L, Zhang CP, Li B, Wen J, Gao W, Sun S, Lv F, Tian $\mathrm{H}$, Tuomilehto J, et al: Increasing prevalence of gestational diabetes mellitus in Chinese women from 1999 to 2008. Diabet Med 28: 652-657, 2011.

7. Leng J, Shao P, Zhang C, Tian H, Zhang F, Zhang S, Dong L, Li L, Yu Z, Chan JC, et al: Prevalence of gestational diabetes mellitus and its risk factors in Chinese pregnant women: A prospective population-based study in Tianjin, China. PLoS One 10: e0121029, 2015.

8. Cho NH, Whitng D, Forouhi N, et al: IDF diabetes atlas, 8th edn. Brussels: Internatonal Diabetes Federaton 2017.

9. Mitanchez D, Yzydorczy C, Siddeek B, Boubred F, Benahmed M and Simeoni U: The offspring of the diabetic mother-short- and long-term implications. Best Pract Res Clin Obstet Gynaecol 29: 256-269, 2015.

10. Bowers K, Laughon SK, Kiely M, Brite J, Chen Z and Zhang C: Gestational diabetes, pre-pregnancy obesity and pregnancy weight gain in relation to excess fetal growth: Variations by race/ethnicity. Diabetologia 56: 1263-1271, 2013.

11. Kristensen P, Susser E, Irgens LM, Mehlum IS, Corbett $K$ and Bjerkedal T: The association of high birth weight with intelligence in young adulthood: A cohort study of male siblings. Am J Epidemiol 180: 876-884, 2014.

12. Tieu J, McPhee AJ, Crowther CA and Middleton P: Screening and subsequent management for gestational diabetes for improving maternal and infant health. Cochrane Database Syst Rev CD007222, 2014.

13. Kung JT, Colognori D and Lee JT: Long noncoding RNAs: Past, present, and future. Genetics 193: 651-669, 2013. 
14. Wang KC and Chang HY: Molecular mechanisms of long noncoding RNAs. Mol Cell 43: 904-914, 2011.

15. Sanchez Y and Huarte M: Long non-coding RNAs: Challenges for diagnosis and therapies. Nucleic Acid Ther 23: 15-20, 2013.

16. Wapinski $\mathrm{O}$ and Chang HY: Long noncoding RNAs and human disease. Trends Cell Biol 21: 354-361, 2011.

17. $\mathrm{Li} \mathrm{CH}$ and Chen Y: Targeting long non-coding RNAs in cancers: Progress and prospects. Int J Biochem Cell Biol 45: 1895-1910, 2013.

18. Carter G, Miladinovic B, Patel AA, Deland L, Mastorides S and Patel NA: Circulatng long noncoding RNA GAS5 levels are correlated to prevalence of type 2 diabetes mellitus. BBA Clin 4: 102-107, 2015.

19. Xie X, Tang B, Xiao YF, Xie R, Li BS, Dong H, Zhou JY and Yang SM: Long non-coding RNAs in colorectal cancer. Oncotarget 7: 5226-5239, 2016.

20. Lin SP, Youngson N, Takada S, Seitz H, Reik W, Paulsen M, Cavaille J and Ferguson-Smith AC: Asymmetric regulation of imprinting on the maternal and paternal chromosomes at the Dlk1-Gt12 imprinted cluster on mouse chromosome 12. Nat Genet 35: 97-102, 2003.

21. $\mathrm{Xu} \mathrm{J}$ and $\mathrm{Xu} \mathrm{Y}$ : The lncRNA MEG3 downregulation leads to osteoarthritis progression via miR-16/SMAD7 axis. Cell Biosci 7: 69, 2017.

22. Zhan R, Xu K, Pan J, Xu Q, Xu S and Shen J: Long noncoding RNA MEG3 mediated angiogenesis after cerebral infarction through regulating p53/NOX4 axis. Biochem Biophys Res Commun 490: 700-706, 2017.

23. Qiu GZ, Tian W, Fu HT, Li CP and Liu B: Long noncoding RNA-MEG3 is involved in diabetes mellitus-related microvascular dysfunction. Biochem Biophys Res Commun 471: 135-141, 2016.

24. Zhu X, Wu YB, Zhou J and Kang DM: Upregulation of IncRNA MEG3 promotes hepatic insulin resistance via increasing FoxO1 expression. Biochem Biophys Res Commun 469: 319-325, 2016.

25. Wang N, Zhu Y, Xie M, Wang L, Jin F, Li Y, Yuan Q and De W: Long noncoding RNA Meg3 regulates mafa expression in mouse beta cells by inactivating Rad21, Smc3 or Sin $3 \alpha$. Cell Physiol Biochem 45: 2031-2043, 2018.

26. Livak KJ and Schmittgen TD: Analysis of relative gene expression data using real-time quantitative PCR and the 2(-Delta Delta C(T)) method. Methods 25: 402-408, 2001.

27. Li JH, Liu S, Zhou H, Qu LH and Yang JH: starBase v2.0: Decoding miRNA-ceRNA, miRNA-ncRNA and protein-RNA interaction networks from large-scale CLIP-Seq data. Nucleic Acids Res 42: D92-D97, 2014.
28. Lu J, Wu J, Zhao Z, Wang J and Chen Z: Circulating LncRNA serve as fingerprint for gestational diabetes mellitus associated with risk of macrosomia. Cell Physiol Biochem 48: 1012-1018, 2018.

29. Li Y, Ren M, Zhao Y, Lu X, Wang M, Hu J, Lu G and He S: MicroRNA-26a inhibits proliferation and metastasis of human hepatocellular carcinoma by regulating DNMT3B-MEG3 axis. Oncol Rep 37: 3527-3535, 2017.

30. Zhang Y, Wu H, Wang F, Ye M, Zhu $\mathrm{H}$ and Bu S: Long non-coding RNA MALAT1 expression in pregnant women with gestational diabetes mellitus. Int J Gynaecol Obstet 140: 164-169, 2018.

31. Buchanan TA, Xiang A, Kjos SL and Watanabe R: What is gestatonal diabetes? Diabetes Care 30 (Suppl 2): S105-S111, 2007.

32. American Diabetes Association (ADA): Standards of medical care in diabetes. Diabetes Care 37: 14-80, 2014.

33. Loegl J, Nussbaumer E, Cvitic S, Huppertz B, Desoye G and Hiden U: GDM alters paracrine regulation of feto-placental angiogenesis via the trophoblast. Lab Invest 97: 409-418, 2017.

34. Esteves JV, Yonamine CY, Pinto-Junior DC, Gerlinger-Romero F, Enguita FJ and Machado UF: Diabetes modulates MicroRNAs 29b-3p, 29c-3p, 199a-5p and 532-3p expression in muscle: Possible role in GLUT4 and HK2 repression. Front Endocrinol (Lausanne) 9: 536, 2018.

35. Chavali V, Tyagi SC and Mishra PK: Differential expression of dicer, miRNAs, and inflammatory markers in diabetic Ins2+/Akita hearts. Cell Biochem Biophys 68: 25-35, 2014.

36. Ji L, Brkić J, Liu M, Fu G, Peng C and Wang YL: Placental trophoblast cell differentiation: Physiological regulation and pathological relevance to preeclampsia. Mol Aspects Med 34: 981-1023, 2013

37. Weiss G, Sundl M, Glasner A, Huppertz B and Moser G: The trophoblast plug during early pregnancy: A deeper insight. Histochem Cell Biol 146: 749-756, 2016.

38. Huang L, Li Y, Wang C, Li N, Hou Y, Chang R, Sun M, Wang R, Zhu L and Qiao C: Overexpression of collapsin response mediator protein 1 inhibits human trophoblastcells proliferation, migration, and Invasion. Reprod Sci 26: 954-960, 2019.

39. Li G, Lin L, Wang YL and Yang H: 1,25(OH)2D3 protects trophoblasts against insulin resistance and inflammation via suppressing mTOR signaling. Reprod Sci 26: 223-232, 2019. International (CC BY-NC-ND 4.0) License. 